\title{
CONTRACTED IDEALS AND PURITY FOR RING EXTENSIONS
}

\author{
J. W. BREWER AND D. L. COSTA
}

\begin{abstract}
In this paper an example is given of a pair of commutative noetherian rings $R \subseteq S$ with $S$ a finite $R$-module and $I S \cap R=I$ for each ideal $I$ of $R$, but having the property that $0 \rightarrow R \rightarrow S$ is not a pure sequence of $R$-modules. Purity of the sequence $0 \rightarrow R \rightarrow S$ is equivalent to $R[X]$ being "ideally closed" in $S[X], X$ an indeterminate. Therefore, the example renders appealing the proposition that for $R$ noetherian and $S$ a noetherian torsion-free $R$-algebra containing $R$, if $a S \cap R=\alpha R$ for each non-zero-divisor a $\epsilon R$, then the extension $R[X] \subseteq S[X]$ has the same properties. Finally, it is also shown that for $R$ noetherian and $0 \rightarrow R \rightarrow$ $S$ pure, with $S$ an $R$-algebra, then $R\left[\left[X_{1}, \ldots, X_{n}\right]\right]$ is pure in $S\left[\left[X_{1}, \ldots, X_{n}\right]\right]$ for each positive integer $n$.
\end{abstract}

Let $S$ be a ring extension of $R$ with $R \subseteq S$. If $X$ is an indeterminate, this note is concerned with the passage to the extension $R[X] \subseteq S[X]$ of certain properties of the extension $R \subseteq S$. Our motivation was the following problem: Suppose that $R$ is noetherian. If for each ideal $I$ of $R, I S \cap R=I$, does $R[X] \subseteq S[X]$ have the same property? Without the noetherian assumption, this question was known to have a negative answer and as we shall soon see, the answer is also negative for $R$ noetherian. Happily, we salvage some positive results, the principal one being: Suppose that $R$ is noetherian and that $S$ is a torsion-free $R$-module. Then $S[X]$ is a torsion-free $R[X]$ -

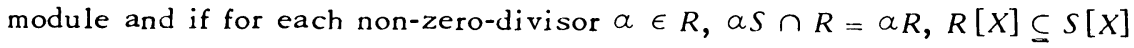
has the same property. The paper concludes with a brief treatment of these questions for the extension $R[[X]] \subseteq S[[X]]$.

We confine ourselves exclusively to commutative unitary rings, and for the most part we restrict our attention to noetherian rings. Our terminology is essentially that of [3].

It is convenient at this point to introduce the notion of purity for $R$ modules. An exact sequence $0 \rightarrow E \rightarrow F$ of $R$-modules is called a pure sequence if for each $R$-module $G, 0 \rightarrow E \otimes G \rightarrow F \otimes G$ is exact. In our case, if $0 \rightarrow R \rightarrow S$ is a pure sequence we shall say that $R$ is pure in $S$ or that $S$ is a pure extension of $R$. From Cohn's well-known solvability of linear equations criterion for purity [6, p. 65, Theorem 3.44], it follows immediately that if $R$ is pure in $S$, then $I S \cap R=I$, for each ideal $I$ of $R$. However,

Received by the editors October 17, 1974.

AMS (MOS) subject classifications (1970)。 Primary 13B25. 
Enochs has given an example to show that the two conditions are not, in general, equivalent. His example appears in [1] and is mentioned in [2]. It is obvious from elementary properties of the tensor product that if $R$ is pure in $S$, then $R[X]$ is pure in $S[X]$. Moreover, one of the key ideas occurring in [1] is the observation that $R$ is pure in $S$ if and only if for each positive integer $n$ and each ideal $I$ of $R\left[X_{1}, \ldots, X_{n}\right]$, the ideal $I S\left[X_{1}, \ldots, X_{n}\right] \cap$ $R\left[X_{1}, \ldots, X_{n}\right]=I$, where $X_{1}, \ldots, X_{n}$ are indeterminates. Thus, by Hilbert's basis theorem for $R$ noetherian, the condition $I S \cap R=I$ for each ideal $I$ of $R$ implies the same condition for $R[X] \subseteq S[X]$ if and only if $I S \cap R=I$ for each ideal $I$ of $R$ implies $R$ is pure in $S$. The following example, suggested to us by E. L. Lady, shows that neither of these implications is valid.

Example 1. An artinian local ring $R$ and an $R$-algebra $S$ containing $R$, finite as an $R$-module, such that $I S \cap R=I$ for each ideal $I$ of $R$, but $R$ is not pure in $S$.

Let $K$ be a finite field with $q$ elements. Set

and

$$
R=K[X, Y] /\left(X^{q+1}, Y^{2}, X^{q} Y\right)=K[x, y]
$$

$$
S=R[T] /\left(T^{2}, x T-x^{q}, y T\right)=R[t]
$$

Claim 1. $R \subseteq S$.

First of all, notice that $S=(R \oplus(R / y R) t) /\left(\left(-x^{q}, x t\right)\right)$, where $R \oplus(R / y R) t \simeq$ the idealization of the $R$-module $R / y R[5, \mathrm{p} .2]$. Now, to see that $R \subseteq S$, we need to see that $\left(\left(-x^{q}, x t\right)\right) \cap R=(0)$. Thus, let $\alpha=(f(x, y), g(x) t)\left(-x^{q}, x t\right) \epsilon$ $R$. Then $a=\left(-x^{q} f(x, y),-x^{q} g(x) t+x f(x, y) t\right)$. Suppose that $f(x, y)=a+m$, $a \in K, m \in(x, y) R$, the unique maximal ideal of $R$. $x^{q} m=0$, so $-x^{q} f(x, y)=$ - $a x^{q}$. Thus, if $a=0$ and $a \in R, a=0$. If $a \neq 0$ and $g(x)=b+x h(x)$, then

$$
\beta=-x^{q} g(x) t+x f(x, y) t=-b x^{q} t+a x t+x^{2}\left(f_{1}(x)\right) t .
$$

But $(R / y R) t \simeq K[X] /\left(X^{q+1}\right)$ and since $a \neq 0, \beta \neq 0$. Thus, $\alpha \notin R$.

Claim 2. $R$ is not pure in $S$.

Now $t \in S$ and is a solution of the system of linear equations

$$
x T=x^{q}, \quad y T=0 .
$$

This system has no solution in $R$, for suppose

$$
\begin{aligned}
f(x, y)= & a+b y+c_{1} x+c_{2} x^{2}+\cdots+c_{q^{x^{q}}} \\
& +d_{1} x y+d_{2} x^{2} y+\cdots+d_{q-1} x^{q-1} y
\end{aligned}
$$

is a solution. Then

$$
\begin{aligned}
x^{q} & =x f(x, y) \\
& =a x+b x y+c_{1} x^{2}+\cdots+c_{q-1} x^{q}+d_{1} x^{2} y+\cdots+d_{q-2} x^{q-1} y,
\end{aligned}
$$


and therefore $a=b=c_{1}=\ldots=c_{q-2}=d_{1}=\ldots=d_{q-2}=0, c_{q-1}=1$. Thus,

But then $y f(x, y)=x^{q-1} y \neq 0$.

$$
f(x, y)=x^{q-1}+c_{q} x^{q}+d_{q-1} x^{q-1} y .
$$

Claim 3. For each ideal $I$ of $R, I S \cap R=I$.

It suffices to show that $(a x+b y) t \in(a x+b y) R$ for all $a, b \in R$. But $(a x+b y) t=a x t=a x^{q}$ and if $a=c+m, c \in K, m \in(x, y)$, then $a x^{q}=c x^{q}=$ $c^{q} x^{q}=a^{q} x^{q}=(a x+b y)^{q}$.

Claim 4. There exist principal ideals of $R\left[Z_{1}, Z_{2}\right]$ which are not contracted ideals from $S\left[Z_{1}, Z_{2}\right], Z_{1}, Z_{2}$ indeterminates.

Since

$$
\begin{aligned}
& t\left(x Z_{1}+y Z_{2}\right)=t x Z_{1}+t y Z_{2}=x^{q} Z_{1} \\
& \quad x^{q} Z_{1} \in\left(\left(x Z_{1}+y Z_{2}\right) s\left[Z_{1}, Z_{2}\right]\right) \cap R\left[Z_{1}, Z_{2}\right] .
\end{aligned}
$$

Suppose that $x^{q} Z_{1} \in\left(x Z_{1}+y Z_{2}\right) R\left[Z_{1}, Z_{2}\right]$, say $x^{q} Z_{1}=\left(F_{0}+F_{1}+\ldots+F_{m}\right)$ - $\left(x Z_{1}+y Z_{2}\right), F_{i}$ a form of degree $i$ in $R\left[Z_{1}, Z_{2}\right]$. Clearly, we must have $x^{q} Z_{1}=F_{0}\left(x Z_{1}+y Z_{2}\right)$. But then $F_{0} x=x^{q}$ and $F_{0} y=0$; that is, $F_{0}$ is a solution in $R$ to the system $(*)$ of Claim 2. This was seen to be impossible.

We would like now to prove the result mentioned in the introduction so we require some terminology. An $R$-module $E$ is called torsion-free if for each $r \in R, e \in E$, re $=0$ implies $r$ is a zero-divisor of $R$ or $e=0$. The condition that $R \subseteq S$ be a torsion-free ring extension is merely the condition that the total quotient ring of $R$ is contained in the total quotient ring of $S$. We can now state our main theorem. Although not as strong as one would like, Claim 4 of the above example shows that it is the best possible.

Theorem 1. Suppose that $R$ is noetherian or an integral domain and that $S$ is a torsion-free $R$-algebra, $S \supseteq R$. Then:

(i) $S[X]$ is a torsion-free $R[X]$-module.

(ii) If $\alpha S \cap R=\alpha R$ for each non-zero-divisor $a \in R$, then $f S[X] \cap$ $R[X]=f R[X]$ for each non-zero-divisor $f \in R[X]$.

Proof. Before giving a proof which treats both cases simultaneously, we give an argument in the domain case which the reader would probably have discovered for himself.

Let $f \in R[X]$ be such that $f$ is a zero-divisor in $S[X]$. Then there exists $d \in S, d \neq 0$ such that $d f=0$. Since $R$ is a domain and $S$ is torsion-free, $f=0$. This proves (i). For (ii), let $f \in R[X]$ with $h \in f S[X] \cap R[X]$. Then $h=f g, g \in S[X]$. Assume the notation is such that

$$
\left(a_{m} X^{m}+a_{m+1} X^{m+1}+\cdots\right)\left(b_{0}+b_{1} X+\cdots\right)=c_{0}+c_{1} X+\cdots,
$$

$$
\text { where } a_{i}, c_{i} \in R, b_{i} \in S, a_{m} \neq 0
$$


and $j$ is the smallest index so that $b_{j} \notin R$. Then $a_{m} b_{j}+a_{m+1} b_{j-1}+\ldots$ $c_{m+j}$ and so $a_{m} b_{j} \in a_{m} S \cap R=a_{m} R$. Therefore, $a_{m} b_{j}=a_{m} b_{j}^{\prime}, b_{j}^{\prime} \in R$. Cancelling $a_{m}$, we reach a contradiction and $g \in R[X]$.

(i) We have proved (i) when $R$ is a domain, so assume now that $R$ is noetherian. Let $f=\sum_{i=0}^{n} r_{i} X^{i} \in R[X]$ be a non-zero-divisor of $R[X]$. If $Z(R)$ denotes the zero-divisors of $R, Z(R)$ is a finite union of prime ideals of $R$, each of which is the annihilator of a single nonzero element of $R$. It follows that $\left(r_{0}, \ldots, r_{n}\right) \nsubseteq Z(R)$ since the annihilator of $\left(r_{0}, \ldots, r_{n}\right) \ldots(0)$. Choose $r \in\left(r_{0}, \ldots, r_{n}\right) \backslash Z(R)$. If $f$ is a zero-divisor in $S[X]$, then for some nonzero $s \in S$, sf and hence $s r=0$. This is impossible.

(ii) Now suppose that $f g=h \in f S[X] \cap R[X], f, h \in R[X], g \in S[X], f$ a non-zero-divisor. Write $f=\sum_{i=0}^{l} r_{i} X^{i}, g=\sum_{i=0}^{m} s_{i} X^{i}, h=\sum_{i=0}^{n} t_{i} X^{i}$, where $n==$ $l+m$, adding zero coefficients for $t$ 's if necessary. Then the equation $f g \cdots h$ is equivalent to the system

$$
\sum_{i+j=k} r_{i} s_{j}=t_{k}, \quad 0 \leq k \leq n .
$$

Since $f$ is a non-zero-divisor in $S[X], g$ is the unique solution of $h=f g$, and so $\left(s_{0}, \ldots, s_{m}\right)$ is the unique solution in $S$ to $(*)$. Therefore, the homogeneous system associated with $(*)$ has no nontrivial solution. By McCoy's theorem [3, p. 147], the ideal $I$ of $R$ generated by the $(m+1) \times(m+1)$ subdeterminants of the coefficient matrix of (*) has annihilator 0 . When $R$ is a domain, one of those determinants is nonzero, say $d$. By Cramer's rule, $d s_{j} \epsilon$ $R$ for each $j$, so $s_{j} \in R$ for each $j$ and $g \in R[X]$. When $R$ is noetherian, $I$ contains a regular element $d \in R$. Again $d s_{j} \in R$ for each $j$ and so $g \in R[X]$.

Note that (ii) can be interpreted as saying that for $R$ noetherian and $R$ $\subseteq S$ a torsion-free extension, $S \cap$ (total quotient ring of $R$ ) $=R$ passes to the polynomial extension $R[X] \subseteq S[X]$.

Note also that some hypothesis on $R$ is required for (i). To see this, notice that Exercises 6 and 7 of [3, p. 62] give a ring $R$ for which (a) nonzero-divisors are units and (b) there exist $p, q \in R$ such that the annihilator of $(p, q)=(0),(p, q) \neq R$. Set $S=R[Y] /(p Y, q Y)$. Then $R \subseteq S, S$ is torsionfree over $R, p+q X$ is a non-zero-divisor in $R[X]$, but in $S[X], y(p+q X)=$ 0 . Here, $y$ denotes the image of $Y$ in $S$ and clearly, $y \neq 0$.

Recall from [3] that the extension $R \subseteq S$ has (LO) for "lying over" if for each prime ideal $P$ of $R$ there exists a prime ideal $P^{\prime}$ of $S$ such that $P^{0} \cap R=P$. This is clearly equivalent to the condition that for each prime ideal $P$ of $R, P S \cap R=P$. We are going to prove that no finiteness assumptions whatsoever are required to insure that $P S \cap R=P$, for each prime ideal $P$ of $R$ passes to polynomial extension. However, as Example 1 shows, even in case $R$ is noetherian, knowing that $I S \cap R=I$ for each ideal $I$ of $R$ does not insure that for each irreducible ideal $J$ of $R[X], J S[X] \cap R\left[X^{\prime}\right]=J$. This is because in the noetherian case knowing that irreducible ideals of 
$R[X]$ are contracted from $S[X]$ is sufficient to guarantee that all ideals are contracted.

Proposition (cf. McAdam [4, p. 708, Proposition 1]). If $R \subseteq S$ has (LO), then $R[X] \subseteq S[X]$ has $(L O)$.

Proof. Let $P^{\prime}$ be a prime ideal of $R[X], P=P^{\prime} \cap R$, and $Q$ a prime ideal of $S$ lying over $P$. If $P^{\prime}=P[X]$, then $Q[X]$ lies over it. Otherwise, consider $\bar{P}^{\prime}$ in $(R / P)[X]$. Set $D=R / P, D_{1}=S / Q, K=$ quotient field of $D$, $L=$ quotient field of $D_{1}$. Then $\bar{P}^{\prime}$ is a height one prime of $D[X]$ and $\bar{P}^{\prime} \cap$ $D=(0)$. Therefore $\bar{P}^{\prime} K[X]$ is a nonzero prime of $K[X]$, say generated by $f$. It follows that $f L[X]$ is contained in a prime ideal $M_{1}$ of $L[X]$ and that $M_{1} \cap K[X]=\bar{P}^{\prime} K[X]$, so $M_{1} \cap D[X]=\bar{P}^{\prime}$. Let $\bar{Q}^{\prime}=M_{1} \cap D_{1}[X]$. Then $\bar{Q}^{\prime} \cap$ $D[X]=\bar{P}^{\prime}$ and with $Q^{\prime}=$ preimage of $Q^{\prime}$ in $S[X]$, we have $Q^{\prime} \cap R[X]=P^{\prime}$.

We now turn to the power series extension $R[[X]] \subseteq S[[X]]$. The fact that $R$ pure in $S$ implies $R[X]$ is pure in $S[X]$ is, as noted previously, merely a "change of rings" theorem. This technique does not apply to $R[[X]]$, and it is an open question whether $R$ pure in $S$ implies $R[[X]]$ pure in $S[[X]]$. We are able to settle the noetherian case and for this we require a a lemma.

For $\left\{X_{a}\right\}$ a collection of indeterminates, denote by $X$ the ideal of $R\left[\left[\left\{X_{a}\right\}\right]\right]$ generated by the $X_{\alpha}$ 's. Inspired by $[2, \mathrm{p} .764$, Theorem 8$]$ we have

Lemma. Suppose that $R \subseteq S$. Then $R$ is pure in $S$ if and only if for each finite family $\left\{X_{i}\right\}$ of indeterminates and for each ideal $I$ of $R\left[\left[\left\{X_{i}\right\}\right]\right]$, the ideal IS $\left[\left[\left\{X_{i}\right\}\right]\right] \cap R\left[\left[\left\{X_{i}\right\}\right]\right] \subseteq \bigcap_{k=0}^{\infty}\left(I+X^{k}\right)$.

Proof. Let $r_{i j} \in R, r_{j} \in R, s_{i} \in S$ with $\sum_{i=1}^{n} r_{i j} s_{i}=r_{j}, j=1, \ldots, m$. Let $X_{1}, \ldots, X_{m}$ be indeterminates and set $f_{i}=\sum_{j=1}^{m} r_{i j} X_{j}$ for $1 \leq i \leq n$ and $f=$ $\sum_{j=1}^{m} r_{j} X_{j}$. Then $f=\sum_{j=1}^{n} s_{i} f_{i}$. Set $I=\left(f_{1}, \ldots, f_{n}\right) R\left[\left[X_{1}, \ldots, X_{n}\right]\right]$. Thus,

$$
f \in I S\left[\left[X_{1}, \ldots, X_{n}\right]\right] \cap R\left[\left[X_{1}, \ldots, X_{n}\right]\right] \subseteq \bigcap_{k=0}^{\infty}\left(I+X^{k}\right) \subseteq I+X^{2}
$$

and so $f=\sum_{i=1}^{n} g_{i} f_{i}+h, h \in \mathbf{X}^{2}$. But $f$ is a form of degree 1 , hence $f=$ $\sum_{i=1}^{n} g_{i}(0) f_{i}$. It follows that $\sum_{i=1}^{n} r_{i j} g_{i}(0)=r_{j}, 1 \leq j \leq m$, and by $[6$, p. 65 , Theorem 3.44] $R$ is pure in $S$.

The converse is proved in [2].

Note that it follows from Example 1 and the above Lemma that $I S \cap R=$ $I$ for each ideal $I$ of $R$ does not pass to the power series extension $R[[X]] \subseteq S[[X]]$, even for $R$ noetherian.

Theorem 2. Let $R \subseteq S$ with $R$ noetherian and $R$ pure in $S$. Then for each positive integer $n, R\left[\left[X_{1}, \ldots, X_{n}\right]\right]$ is pure in $s\left[\left[X_{1}, \ldots, X_{n}\right]\right]$.

Proof. By induction, it suffices to prove that $R[[X]]$ is pure in $S[[X]]$. 
Let $\left\{X_{i}\right\}$ be a finite set of indeterminates distinct from $X$. In $R\left[\left[X,\left\{X_{i}\right\}\right]\right]$, set $\boldsymbol{X}=\left(X,\left\{X_{i}\right\}\right)$ and $X_{0}=\left(\left\{X_{i}\right\}\right)$. Since $R$ is pure in $S$, by the Lemma, for each ideal $I$ of $R\left[\left[X,\left\{X_{i}\right\}\right]\right]-(R[[X]])\left[\left[\left\{X_{i}\right\}\right]\right]$,

$$
I S\left[\left[X,\left\{X_{i}\right\}\right]\right] \cap R\left[\left[X,\left\{X_{i}\right\}\right]\right] \subseteq \bigcap_{k=0}^{\infty}\left(I+X^{k}\right)=I
$$

since $\left(R\left[\left[X,\left\{X_{i}\right\}\right]\right], X\right)$ is a Zariski ring. But $\bigcap_{k=0}^{\infty}\left(I+X_{0}^{k}\right)=I$ since $\left(R\left[\left[X,\left\{X_{i}\right\}\right]\right], X_{0}\right)$ is also a Zariski ring. By the Lemma, $R[[X]]$ is pure in $S[[X]]$.

Added in proof. The question "Does $R$ pure in $S$ imply $R[[X]]$ pure in $S[[X]]$ " has been settled in the negative.

\section{REFERENCES}

1. E. Enochs, On absolutely pure modules (preprint).

2. R. W. Gilmer, Jr. and J. Mott, Some results on contracted ideals, Duke Math. J. 37 (1970), 751-767. MR $42 \# 3067$.

3. I. Kaplansky, Commutative rings, Allyn and Bacon, Boston, Mass., 1970. MR $40 \# 7234$.

4. S. McAdam, Going down in polynomial rings, Canad. J. Math. 23 (1971), 704-711. MR 43 \#6202.

5. M. Nagata, Local rings, Interscience Tracts in Pure and Appl. Math., no. 13, Interscience, New York, 1962. MR 27 \#5790.

6. J. J. Rotman, Notes on homological algebra, Van Nostrand Reinhold Math. Studies, no. 26, Van Nostrand, New York, 1970.

DEPARTMENT OF MATHEMATICS, UNIVERSITY OF KANSAS, LAWRENCE, KANSAS 66045

DEPARTMENT OF MATHEMATICS, UNIVERSITY OF VIRGINIA, CHARLOTTESVILLE, VIRGINIA 22903 